\title{
Monseigneur Elzéar-Alexandre Taschereau et la création du diocèse de Nicolet
}

\section{Nive Voisine}

Volume 52, 1985

URI : https://id.erudit.org/iderudit/1007001ar

DOI : https://doi.org/10.7202/1007001ar

Aller au sommaire du numéro

Éditeur(s)

Les Éditions Historia Ecclesiæ Catholicæ Canadensis Inc.

ISSN

0318-6172 (imprimé)

1927-7067 (numérique)

Découvrir la revue

Citer cet article

Voisine, N. (1985). Monseigneur Elzéar-Alexandre Taschereau et la création du diocèse de Nicolet. Sessions d'étude - Société canadienne d'histoire de l'Église catholique, 52, 35-50. https://doi.org/10.7202/1007001ar
Résumé de l'article

$\mathrm{M}^{\mathrm{gr}}$ Elzéar-Alexandre Taschereau, archevêque de Québec, est un des principaux partisans de la création du diocèse de Nicolet. Lors de la tentative avortée de 1875-1878, il se prononce ouvertement en faveur de la mesure, mais sans faire de pressions spéciales à Rome. Mais à partir de 1882, il prend la tête du mouvement en faveur de Nicolet, écrit régulièrement mémoires et lettres à la Propagande, insiste sur la nécessité d'agir rapidement, conseille les pétitionnaires, rameute une majorité de membres de l'épiscopat. Bien plus, au moment où le commissaire apostolique, dom Henri Smeulders, se prononce contre le diocèse de Nicolet, $\mathrm{M}^{\mathrm{gr}}$ Taschereau va à Rome piloter les dossiers et faire pression sur les membres de la Congrégation de la Propagande pour arracher une décision définitive et favorable à sa cause. Ce qu'il obtient à l'arraché le 30 septembre 1884 et qui fait dire à un autre chaud partisan de Nicolet, $\mathrm{M}^{\mathrm{gr}}$ Calixte Marquis : «Je n'ai pas fait le diocèse, je l'ai fait faire par Son Éminence le cardinal Taschereau, grand ami de ma jeunesse ». L'archevêque de Québec intervient si fermement et si instamment parce que le problème de la création du diocèse de Nicolet est directement relié aux autres débats de la période comme la question universitaire, le problème de l'« influence indue », les animosités entre les autorités diocésaines de Québec et les intransigeants de la province.
Tous droits réservés (C) Les Éditions Historia Ecclesiæ Catholicæ Canadensis Inc., 1985
Ce document est protégé par la loi sur le droit d'auteur. L’utilisation des services d'Érudit (y compris la reproduction) est assujettie à sa politique d'utilisation que vous pouvez consulter en ligne.

https://apropos.erudit.org/fr/usagers/politique-dutilisation/ 


\title{
Monseigneur Elzéar-Alexandre Taschereau et la création du diocèse de Nicolet
}

\author{
Nive VoISINE \\ Département d'histoire \\ Université Laval, Québec
}

\section{RÉSUMÉ}

$M^{g r}$ Elzéar-Alexandre Taschereau, archevêque de Québec, est un des principaux partisans de la création du diocèse de Nicolet. Lors de la tentative avortée de 1875-1878, il se prononce ouvertement en faveur de la mesure, mais sans faire de pressions spéciales à Rome. Mais à partir de 1882, il prend la tête du mouvement en faveur de Nicolet, écrit régulièrement mémoires et lettres à la Propagande, insiste sur la nécessité d'agir rapidement, conseille les pétitionnaires, rameute une majorité de membres de l'épiscopat. Bien plus, au moment où le commissaire apostolique, dom Henri Smeulders, se prononce contre le diocèse de Nicolet, $M^{g r}$ Taschereau va à Rome piloter les dossiers et faire pression sur les membres de la Congrégation de la Propagande pour arracher une décision définitive et favorable à sa cause. Ce qu'il obtient à l'arraché le 30 septembre 1884 et qui fait dire à un autre chaud partisan de Nicolet, $M^{g r}$ Calixte Marquis: "Je n'ai pas fait le diocèse, je l'ai fait faire par Son Éminence le cardinal Taschereau, grand ami de ma jeunesse». L'archevêque de Québec intervient si fermement et si instamment parce que le problème de la création du diocèse de Nicolet est directement relié aux autres débats de la période comme la question universitaire, le problème de l' «influence indue», les animosités entre les autorités diocésaines de Québec et les intransigeants de la province. 
Bien des personnes ont contribué à la création du diocèse de Nicolet: les prêtres du Collège qui ont été parmi les premiers à en avoir l'idée et qui y ont vu une question de survie pour leur institution, l'abbé JosephAntoine-Irenée Douville, entre autres, jouant un important rôle de coordonnateur; l'abbé (puis Monseigneur) Calixte Marquis dont on a déjà rappelé ici le travail infatigable et efficace'; à Québec, les professeurs et partisans de l'Université Laval et les familiers de l'archevêque qui ont pris l'affaire en main et l'ont menée à terme; $\mathrm{M}^{\mathrm{gr}}$ Dominique Racine qui a détendu le dossier à Rome; et combien de colliaborateurs plus discretets où passagers...

Sans vouloir enlever à quiconque son mérite, je vais m'attacher à décrire le rôle primordial joué, tout au long des années, par l'archevêque de Québec, $\mathrm{M}^{\mathrm{gr}}$ Elzéar-Alexandre Taschereau. Une phrase de Calixte Marquis lui-même m'y invite: «Je n'ai pas fait le diocèse, disait-il, je l'ai fait faire par Son Éminence le cardinal Taschereau, grand ami de ma jeunesse». Que se cache-t-il de vérité derrière cette boutade de celui qui en a commis bien d'autres? Telle est lá question qui m'incitc à relire dans une perspective plus étroite la masse des documents des archives romaines et canadiennes et à les interroger sur les faits et gestes du prélat québécois.

À prime abord, il m'apparaît que l'intervention de $\mathrm{M}^{\mathrm{gr}}$ Taschereau a été déterminante et qu'elle a, à un certain moment, éclipsé toutes les autres. C'est ce que je vais essayer de confirmer, non pas en résumant une nouvelle fois les péripéties nombreuses de la longue marche vers la création du diocèse ${ }^{2}$, mais en analysant les divers appuis et les initiatives de celui qu'on appelait parfois dans la correspondance «Quelqu'un» ou «Qui de droit».

\section{1- Sympathie et appuis}

Quand le projet de la création d'un diocèse à Nicolet est lancé, $\mathrm{M}^{\mathrm{gr}}$ Taschereau est archevêque depuis à peine quatre ans et il a affronté des situations difficiles: la division à propos du Programme catholique, les difficultés entre $\mathrm{M}^{\mathrm{gr}}$ Bourget et les Sulpiciens, les attaques contre l'Université Laval, la mise en question du rôle des prêtres en politique. En1875, plusieurs de ces problèmes sont toujours débattus au Québec ou à Rome.

\footnotetext{
${ }^{1}$ Alphonse Roux, «Monseigneur Calixte Marquis et l'érection du diocèse de Nicolet", Société canadienne d'histoire de l'Église catholique, Figures nicolétaines, Congrès de Nicolet, 1944, pp. 29-83.

2 Voir Nive Voisine, «La création du diocèse de Nicolet (1885)», Les Cahiers Nicolétains, V, 1(mars 1983), pp. 3-41; VI, 4(décembre 1984), pp. 147-214.
} 
Dans presque chaque cas, l'archevêque retrouve $\mathrm{M}^{\mathrm{gr}}$ Laflèche de TroisRivières parmi ses antagonistes. Il est donc prêt à recevoir avec sympathie tout ce qui peut embarrasser son coriace collègue.

C'est ce qui se produit dès les premiers moments du projet de création du diocèse de Nicolet. Les prêtres du Séminaire de Nicolet insastisfaits de Laflèche et craignant pour leur avenir, un groupe de curés dissidents qui ont soulevé de nouveau la question du dixième (taxe de $10 \%$ sur les revenus des curés et missionnaires) et surtout Calixte Marquis qui a toujours été un trouble-fête pour les évêques, concoctent discrètement une supplique et des mémoires justificatifs en étroite collaboration avec l'archevêque et son entourage. Pendant l'hiver 1875, par exemple, Marquis vient plusieurs fois «avec une cargaison complète de mémoires, suppliques, lettres»; il les présente à Taschereau qui lui conseille «de retrancher les endroits où l'évêque des Trois-Rivières est un peu maltraité $»^{3}$. Le porte-parole de Nicolet lui communique également les documents qui partent pour Rome.

Les familiers et les amis de l'archevêque sont aussi mis dans le coup. Le procureur, Jean-Baptiste-Zacharie Bolduc, rencontre fréquemment Marquis ou correspond avec lui et il sert d'intermédiaire pour renseigner les autres. On contacte Benjamin Pâquet à Rome pour présenter et soutenir la demande auprès de la Propagande et du pape; sa première réaction est plutôt froide: il veut bien s'occuper de cette affaire, mais il ne veut pas paraitre la provoquer et il fait avertir Marquis d'envoyer directement les documents aux diverses instances ${ }^{4}$. Parmi les évêques, $M^{\mathrm{gr}}$ Antoine Racine de Sherbrooke est le seul, avec l'archevêque, à être mis au courant. Tous manifestent leur sympathie et promettent leur appui.

D'abord présentés à Québec, les documents sont acheminés à Rome sur les conseils de l'archevêque, mais la Propagande exige l'opinion de l'épiscopat de la province. Taschereau en avertit ses collègues, y compris Laflèche, et leur fournit la supplique et le mémoire explicatif. Il refuse à l'évêque de Trois-Rivières la liste des signataires et les pièces justificatives; de façon générale, il n'engage aucune discussion avec lui et le réfère plutôt au porte-parole de Nicolet, le curé Louis-Stanislas Malo de Bécancour.

La réunion des évêques a lieu le 23 mars 1876 à Québec et n'en regroupe que cinq, $\mathrm{M}^{\mathrm{grs}}$ Bourget, Fabre et Langevin étant absents. Chacun doit répondre à la question: «est-il opportun de diviser le diocèse des

${ }^{3}$ J.-B.-Z. Bolduc à Benjamin Pâquet, 19 mars 1875, Archives du Séminaire de Québec (désormais ASQ), Université, $111, \mathrm{n}^{\circ} 44$.

${ }^{4}$ B. Pâquet à J.-B.-Z. Bolduc, 4 avril 1875, idib., 35, nº 45 . 
Trois-Rivières et de faire l'érection d'un autre diocèse dont le siège épiscopal soit à Nicolet?» Laflèche, Thomas Duhamel d'Ottawa et, par lettres, Ignace Bourget et Édouard-Charles Fabre de Montréal se prononcent clairement contre Nicolet. $\mathrm{M}^{\mathrm{gr}}$ Louis-Zéphirin Moreau de Saint-Hyacinthe «ne voit pas qu'il soit possible actuellement d'ériger à Nicolet un nouveau diocèse»; des lettres adressées à Laflèche prouvent qu'il est très opposé au projet. $\mathrm{M}^{\mathrm{gr}}$ Antoine Racine de Sherbrooke se dit favorable à la division «si elle est possible ni injuste envers les créanciers de la Corporation épisco-

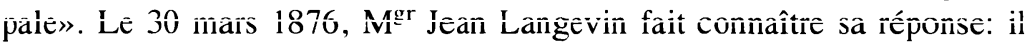
croit le démembrement possible, opportun ou désirable, mais non urgent; il conclut: «bien que je pense ce démembrement absolument praticable et désirable sous certains rapports, je le trouverais prématuré pour le moment $»^{5}$.

Pour sa part, $\mathrm{M}^{\mathrm{gr}}$ Taschereau donne le ton à la discussion en se prononçant le premier «pour la possibilité et l'opportunité» de la création d'un nouveau diocèse. Il s'en explique plus longuement dans la lettre au cardinal Franchi où il rapporte le résultat de la réunion des évêques. Il souligne l'opposition de $\mathrm{M}^{\mathrm{gr}}$ Laflèche, mais ne l'explicite pas, car, dit-il, l'évêque de Trois-Rivières fera connaître lui-même ses objections; il résume en quelques mots la position de chacun des prélats et transcrit en latin la lettre de Langevin; il donne cependant une place spéciale à $\mathrm{M}^{\mathrm{gr}}$ Moreau qui, dit-il, «pense que l'érection d'un nouveau diocèse n'est pas possible dans les circonstances actuelles, mais qu'elle lui apparaît très souhaitable», ce qui dépasse l'opinion de l'évêque de Saint-Hyacinthe. L'archevêque consacre, cependant, la plus grande partie de sa longue lettre à réfuter les arguments de Laflèche et à expliquer les raisons favorables à Nicolet. Il attribue une opinion semblable à $\mathrm{M}^{\mathrm{gr}} \mathrm{A}$. Racine - il commence chacun de ses arguments par: «L'archevêque de Québec et l'évêque de Sherbrooke pensent que...» - et il conclut de façon très claire: «le démembrement immédiat m'apparaît nécessaire et possible» ${ }^{6}$. L'archevêque interviendra personnellement assez peu par la suite, mais son entourage de l'archevêché et de l'université fait peser tout son poids en faveur de Nicolet et assure un lobbying continuel à Rome. L'un d'entre eux, le procureur Bolduc, le décrit ainsi:

\footnotetext{
${ }^{5} \mathrm{M}^{\mathrm{gr}}$ A. Racine, [Procès-verbal de l'assemblée des évêques tenue à Québec le 23 mars 1876], Archives de l'Archevêché de Québec (désormais AAQ), $10 \mathrm{CP}$, Épiscopat du Québec, I, pp. 52-53; $\mathrm{M}^{\mathrm{gr}} \mathrm{J}$. Langevin à $\mathrm{M}^{\mathrm{gr}}$ Taschereau, 30 mars 1876, Archives de l'archevêché de Rimouski, Corr. spéciale, I, 137.

${ }^{6} \mathrm{M}^{\mathrm{gr}}$ Taschereau au card. Franchi, 12 avril 1876, Archives de.Propaganda Fide à Rome (désormais APFR), SRC-ASC, 14(1876), f.226r-231v. La lettre, en latin, n'a pas été copiée dans les registres et l'archevêque a toujours refusé d'en communiquer une copie à Laflèche.
} 
Il y a plusieurs gros bonnets à Rome qui travaillent pour le succès de cette affaire. Moi, Je n'ai pas un gros bonnet ni même une grosse tête et pourtant, j'ai eu assez de toupet pour faire un mémoire sur cette question.

J'ai recueilli tous les arguments que les autres n'ont pas voulu donner.

La Propagande aura de la difficulté à refuser votre demande. ${ }^{7}$

L'effet ne tarde pas à se faire sentir puisque, le 24 mai 1876, le préfet de la Propagande écrit à Laflèche que l'archevêque et trois suffragants («tres Episcopi suffraganii») ont déclaré la division du diocèse «non seulement utile mais également nécessaire» («manifestaverint propositam divisionem non modo utilem sed etiam necessariam esse») ${ }^{8}$. Alors que le débat se déplace à Rome, Laflèche se dit renversé par l'interprétation qu'on y donne de l'assemblée du 23 mars et il dénonce Taschereau:

Votre Éminence me permettra de lui dire aujourd'hui que toute cette affaire, dans mon humble opinion, est surtout l'oeuvre de $\mathrm{M}^{\mathrm{gr}}$ l'Archevêque par l'encouragement et l'aide qu'il a donnés aux Chefs de ce mouvement. Pour quels motifs en a-t-il agi ainsi? Que Votre Éminence me dispense de les scruter: c'est une affaire trop délicate et qui demanderait un trop long exposé. ${ }^{9}$

Il continuera à le faire lors de son voyage à Rome et auprès du délégué apostolique, $\mathrm{M}^{\mathrm{gr}}$ George Conroy, jusqu'à ce qu'il obtienne une décision favorable le 6 avril 1878 .

\section{2- Dénonciations de Laflèche}

Si le passage de $\mathrm{M}^{\mathrm{gr}}$ Conroy réussit à mettre en veilleuse la question de la division du diocèse de Trois-Rivières - Laflèche peut même croire qu'elle est réglée pour très longtemps - il n'en est pas de même pour la plupart des autres problèmes qui reviennent à la surface avant même le départ et la mort du délégué. Dans les années qui suivent, les discussions entraînent des divisions nouvelles au sein de l'épiscopat du Québec; une constante apparaît: $\mathrm{M}^{\mathrm{gr}}$ Laflèche est presque toujours à l'origine des débats et, quand il ne l'est pas, les instigateurs le consultent et l'obligent à s'impliquer rapidement face à l'antagoniste par excellence, l'archevêque Taschereau. La riposte québécoise consistera à saper la crédibilité de l'évê-

\footnotetext{
${ }^{7}$ J.-B.-Z. Bolduc à Thomas Caron, 17 mai 1876, Archives du Séminaire de Nicolct (désormais ASN) Succ. T. Caron, Boîte N ${ }^{\circ} 2,7$.

${ }^{8}$ Card. Franchi à $\mathrm{M}^{\mathrm{gr}}$ Laflèche, 24 mai 1876, APFR, Lettere, 372 (1876), f.229v-230r.

${ }^{9} \mathrm{M}^{\mathrm{gr}}$ Laflèche au card. Franchi, 29 juin 1876, Archives de l'évêché de Trois-Rivières (désormais AETR), ibid., SRC-ASC, 15(1877), f.569r-560v.
} 
que de Trois-Rivières et à l'inciter à démissionner de son poste, ce qui serait une bonne manière de régler les problèmes de la rive sud.

De tous les nombreux problèmes qui sont débattus entre 1878 et 1882 . deux, en particulier, conduisent à un affrontement grave et public entre Laflèche et Taschereau: l' «influence spirituelle indue», la question universitaire. La première concerne l'ingérence abusive de certains prêtres dans les élections et avait donné lieu, sur ordres de Rome, à une déclaration épiscopale collective (rédigée par Laflèche) et à des directives très précises au clergé ${ }^{10}$. Fortement mécontents, des groupes de curés de Montréal, Trois-Rivières et Rimouski s'organisent pour dénoncer à Rome le délégué à tendances «libérales», disent-ils, et ses directives qui réduisent au silence les «défenseurs de la vérité». Mais c'est tout spécialement la contestation de l'élection de Berthier, en 1880, qui remet d'actualité l'«influence spirituelle indue»: cinq curés sont cités à la barre, on songe même à y faire témoigner des théologiens et un évêque (Laflèche), les avocats font raconter et analysent les confessions de plusieurs témoins. Les évêques s'inquiètent et sentent le besoin de publier une lettre pastorale collective "sur le respect dû à la parole de Dieu et au sacrement de pénitence», mais ils sont unanimes à ne pas exiger immédiatement le rappel ou même l'amendement de la loi électorale de 1875 , qu'ils avaient déjà réclamés en $1877^{11}$.

$\mathrm{M}^{\mathrm{gr}}$ Laflèche accepte l'opinion majoritaire, même s'il croit personnellement que le temps «le plus opportun» est arrivé pour demander les changements. Néanmoins, sitôt connue l'annulation de l'élection de Berthier «pour cause d'influence spirituelle indue», il publie anonymement une série d'articles sur le sujet dans le Journal des Trois-Rivières et les distribue généreusement, sous forme de brochure, au clergé et aux hommes politiques.

La conclusion résume bien ses idées et sa nouvelle position:

Nous croyons que ce qui précède établit clairement et solidement que la loi de l'influence spirituelle indue, telle qu'interprétée par les tribunaux civils de la province, produit un conflit fort regrettable entre l'Église et l'État, porte une atteinte grave à la liberté religieuse et civile de tous les

10 «Lettre pastorale des évêques de la province ecclésiastique de Québec», 11 oct. 1877. Mandements des évêques de Québec (désormais $M E Q$ ), VI, pp. 51-53; "Circulaire des évêques de la province ecclésiastique de Québec au clergé de ladite province», 11 oct., 1877. ibid., Vl, pp. 44-50.

11 «Lettre pastorale des évêques de la province ecclésiastique de Québec, sur le respect dû à la parole de Dieu et au sacrement de pénitence», I $1^{\text {er }}$ juin 1880, Ibid., VI, pp. 208-214; [Procès-verbal de l'assemblée des évêques tenue à Québec le ler juin 1880]. AAQ. $10 \mathrm{CP}$. Épiscopat du Québec, I, pp. 97-99. 
citoyens, à quelque parti politique, et à quelque croyance religieuse qu'ils appartiennent, et sans aucun avantage pour personne.

En conséquence, nous engageons tous les amis sincères de la véritable liberté, à user de toute leur influence pour faire abroger une loi qui comporte d'aussi funestes conséquences, ou à la faire amender de manière qu'elle ne puisse jamais être appliquée aux choses du domaine religieux et spirituel. ${ }^{12}$

Plusieurs évêques - Langevin, Moreau, Duhamel — lui accordent leur appui, ce qui l'encourage à intervenir auprès des hommes politiques. Il pousse donc le député Michel Mathieu à présenter un projet de loi dans le sens de ses recommandations et, devant les réticences du ministère, il entreprend de convaincre le premier ministre Joseph-Adolphe Chapleau par une série de lettres qui sont presque des mémoires ${ }^{13}$. C'en est trop pour Taschereau qui n'aime guère qu'on lui passe par dessus la tête: il appuie le refus de Chapleau, dénonce à la Propagande les manoeuvres de Laflèche, puis envoie tout le dossier à Rome pour avis immédiat. La réponse constitue un soufflet pour Laflèche. Le cardinal Simeoni rappelle que «certain suffragant de Votre Seigneurie cherche actuellement à recourir au Parlement, pour faire modifier la loi des élections relativement à l'influence dite indue» et il ordonne de

notifier à chacun des suffragants, de la part de Sa Sainteté, que chacun des Prélats individuellement ait à s'abstenir d'agiter soit dans le Parlement, soit dans la presse, la question de la modification de la loi concernant la dite influence indue. Que s'il arrivait une époque où les évêques réunis jugeassent tous ensemble que le temps opportun est venu de faire la susdite demande, ils devront d'abord recourir à cette Sacrée Congrégation pour en recevoir les instructions nécessaires. ${ }^{14}$

Et quand Laflèche se rend à Rome pour s'expliquer, l'archevêque manoeuvre rapidement pour obtenir une nouvelle condamnation de son collègue:

Le Saint-Père, lui écrit Simeoni, a appris avec déplaisir que certains catholiques de votre province cherchent à fomenter encore des discussions, soit par rapport à l'influence indue dans les élections politiques, soit par rapport à la succursale de l'Université Laval établie à Montréal. Pour lever donc tout doute quelconque à ce sujet et pour mettre fin, une

$12\left[\mathrm{M}^{\mathrm{gr}}\right.$ Laflèche], L'influence spirituelle indue devant la liberté religieuse et civile. TroisRivières, Journal des Trois-Rivières, $1881,100 \mathrm{p}$.

$13 \mathrm{M}^{\mathrm{gr}}$ Laflèche à M. Mathieu, 27 avril 1881, AETR, Registre des lettres, VII, 11; le même à J.-A. Chapleau, 24 avril 1881, ibid., VII, 12; le même au même, 22 juillet 1882, ibid., VII. 29.

14 Card. Simeoni à $\mathrm{M}^{\mathrm{gr}}$ Taschereau, 13 sept. 1881, MEQ, VI, pp. 270-272. 
fois pour toutes, aux dissensions susdites, dans l'audience du 22 [décembre] courant. il a de nouveau ordonné à Votre Seigneurie que c'est sa volonté expresse que l'on observe rigoureusement les deux décrets donnés par Sa Sainteté sur les susdites questions. en septembre dernier. Que, du reste, les individus qui se disent défenseurs de Montréal et qui restent encore à Rome, le font contre la volonté du SaintPère, et abusent ainsi des circonstances politiques actuelles. ${ }^{15}$

Il va sans dire que tous ces documents sont publiés dans les journaux par les bons soins de l'archevêque.

Pendant le même temps, Laflèche et Taschereau s'affrontent également à propos de la question universitaire. Sur ordre de Rome, une succursale de l'Université Laval a été ouverte à Montréal en 1878, mais un violent conflit a surgi entre Laval et l'École de médecine et de chirurgie de Montréal. Laflèche ne s'implique pas au début de la querelle, mais les Montréalistes le consultent régulièrement et tout le monde sait qu'il est en faveur d'une université autonome à Montréal. En octobre 1880, il accepte de signer avec tous ses collègues une pétition à la Reine demandant d'interpréter ou d'amender la charte de l'université pour lui permettre d'affirmer clairement son droit d'ouvrir des succursales, mais, en février 1881, malgré une argumentation serrée de l'archevêque, il refuse d'accepter un texte assez semblable envoyé au gouvernement du Québec. Bien plus, après avoir signé avec ses collègues une déclaration «pour mettre fin aux écrits et aux nouvelles dont on se sert pour rendre l'Université Laval odieuse et suspecte, infirmer l'autorité du décret apostolique et empêcher les intentions pacifiques du Saint-Père de se réaliser», il écrit quelques jours plus tard à son métropolitain:

À la suite de perplexités, je dois obéir à la voix de ma conscience, et déclarer à Votre Grandeur que je regrette la signature que j'ai apposée au bas de la déclaration collective des Évêques de la Province au sujet de la plainte de l'Université-Laval, et que par la présente je la retire pour des raisons que je vais exposer au St-Siège. ${ }^{16}$

On peut imaginer l'effet de cette volte-face et, surtout, la colère de l'archevêque qui obtient une première déclaration de Rome le 13 septembre 1881:

Le Saint-Père, ayant mis à l'examen la question soulevée de nouveau au sujet de l'Université Laval et de la succursale établie à Montréal, a

${ }_{15}$ Id. à id., 31 déc. 1881, Le Journal de Québec, 23 janv. 1882, p. 2.

16 [Procès-verbal de l'assemblée des évêques tenue à Québec les 19-20 octobre 1881], AAQ. $10 \mathrm{CP}$, Épiscopat du Québec, I, pp. 107-109; $\mathrm{M}^{\mathrm{gr}}$ Laflèche à $\mathrm{M}^{\mathrm{gr}}$ Taschereau, 26 oct. 1881, Registre des lettres, VII, p. 119. 
ordonné expressément. dans l'audience extraordinaire d'hier, tenue pour traiter uniquement de cette affaire, de signifier à Votre Seigneurie que c'est sa volonté décidée que l'on doit s'en tenir au décret de cette Sacrée Congrégation, émané le premier jour de février 1876, et continuer à y donner exécution.

Sa Sainteté nourrit la confiance que le clergé et le peuple catholique du Canada, dont Elle a toujours reçu les preuves les plus éclatantes de dévouement et d'attachement au Saint-Siège, se conformeront unanimement à ses ordres susdits et que les divers prélats travailleront sans relâche à ramener dans les esprits la concorde et la paix. ${ }^{17}$

Pendant ce temps, à Rome, Laflèche, d'abord vertement reçu par le secrétaire de la Propagande, $\mathrm{M}^{\mathrm{gr}}$ Ignazio Masotti, réussit à se faire entendre par Léon XIII et contre-attaque dans un long et virulent mémoire qui met en cause l'archevêque, son entourage, l'Université Laval et certains officiers de la Propagande ${ }^{18}$. Taschereau riposte, non seulement en publiant sa propre réfutation ${ }^{19}$, mais en faisant intenter de véritables procès à Laflèche par les principaux incriminés, l'Université Laval et le grand vicaire Cyrille-Étienne Légaré, entre autres. Surtout, dans les cercles québécois, s'accrédite de plus en plus l'idée que Laflèche n'est plus sui compos et qu'il faudrait l'obliger à démissionner. On connaît cette page célèbre du journal intime de $\mathrm{M}^{\mathrm{gr}}$ Michel-Édouard Méthot, alors recteur de l'Université Laval, commentant le retrait de la signature de Laflèche:

ce revirement subit de la part d'un Évêque, d'un vieillard est un grand scandale. Les laïques qui, plus que nous autres clercs, ont le sentiment de l'honneur, au moins humain, ne se gênent pas pour dire qu'il est fou! Ce que je sais bien, c'est qu'il appartient à une famille de fous. Que de fois, dans mon enfance, lorsque je passais mes vacances à Stc-Annc Lapérade chez mon excellent oncle, Elzéar Méthot, - quel monde de souvenirs ces mots me réveillent! - j'ai vu passer dans la rue un des frères de $\mathrm{M}^{\mathrm{gr}}$ Laflèche, fou, poursuivi par une bande d'enfants qui en faisaient leur jouet! Et ses soeurs! Lui-même le sait parfaitement: lorsqu'on voulut le faire évêque, il refusa, disant qu'il appartenait à une famille de fous, qu'il pourrait bien devenir fou lui-même, et qu'alors on se repentirait de l'avoir élevé à l'épiscopat. N'a-t-il pas été un peu bien prophète? $?^{20}$

${ }^{17}$ Card. Simeoni à $\mathrm{M}^{\mathrm{gr}}$ Taschereau, 13 sept. 1881, MEQ, VI, pp. 273-274.

18 [ $\mathrm{M}^{\mathrm{gr}}$ Laflèche], Mémoire de l'Évêque des Trois-Rivières sur les difficultés religieuses en Canada, Trois-Rivières, Impr. G. Désilets, 1882, 178 p.

${ }^{19} \mathrm{M}^{\mathrm{gr}}$ Taschereau, Remarques sur le Mémoire de l'Évêque des Trois-Rivières sur les difficultés religieuses en Canada, Rome, I882, Québec, 1882, 88 p.

20 Journal de $M^{g r}$ Michel-Édouard Méthot, 29 oct. 1881, ASQ, Manuscrit 16. 
Taschereau lui-même ne va pas aussi loin, du moins dans les documents officiels, mais il ne cesse de rappeler les contradictions de Laflèche et sa chasse aux sorcières: "L'auteur du Mémoire se pose toujours luimême comme l'unique criterium de la vérité et ne paraît pas soupçonner qu'on puisse différer d'opinion avec lui sans être un libéral, un gallican, un ennemi de l'Église» ${ }^{21}$. Ses amis, les évêques Antoine et Dominique Racine, vont plus loin et demandent, à plusieurs reprises, la démission de l'évêque de Trois-Rivières. Le premier, par exemple, écrit au recteur Thomas-Étienne Hamel de l'Unıversité Laval: «Ce qui est opportun pour $M^{\mathrm{gr}}$ l'Archevêque, c'est de demander au St-Siège qu'il mette un frein à la fureur mitrée ou qu'il lui ôte sa mître administrative»" ${ }^{22}$; il suggère même à l'archevêque de faire signer une pétition pour obtenir cette démission. Son frère Dominique, évêque de Chicoutimi, est du même avis et s'adresse directement à Rome. Le 20 novembre 1981, il écrit au préfet de la Propagande:

Ce mot, que m'écrivait dernièrement un des suffragants, exprime, je le crois du moins, les sentiments de tous les autres Évêques, le voici: «Après tout ce qui vient de se passer, il est bien évident que jamais Monseigneur Laflèche ne pourra s'entendre avec ses confrères, surtout en ce qui concerne de près ou de loin l'Université Laval». S'il doit en être ainsi, Éminence, je me demande, si pour le bien de la paix et de la religion, $\mathrm{M}^{\mathrm{gr}}$ Laflèche ne ferait pas mieux de prendre sa retraite. ${ }^{23}$

Il renouvelle sa demande régulièrement lors de son voyage à Rome en 1882-1883. Les journaux ont rapidement vent de ces démarches et parlent souvent de la possibilité de déposer l'évêque de Trois-Rivières. Aurait-il eu quelque velléité de démissionner, il n'en faut pas plus pour convaincre Laflèche que son devoir est de demeurer en place pour continuer le bon combat. Et alors, pensent les Québécois, la meilleure façon de mater le coriace chef des intransigeants n'est-il pas d'obtenir le démembrement de son diocèse et la nomination à Nicolet d'un évêque qui contrebalancerait son influence au centre du Québec? Consciemment ou non, Taschereau va entrer avec enthousiasme dans ces vues.

\section{3- Prise en mains du problème}

La seconde et dernière tentative de création du diocèse de Nicolet se déroule dans des conditions très différentes de la première et permet à l'ar-

\footnotetext{
${ }^{21} \mathrm{M}^{\mathrm{gr}}$ Taschereau, Remarques, p. 65.

${ }^{22} \mathrm{M}^{\mathrm{g} r}$ A. Racine à T.-E. Hamel, 10 nov. 1881, ASQ, Université 135. B.H.

${ }^{23} \mathrm{M}^{\mathrm{gr}} \mathrm{D}$. Racine au card. Simeoni, 20 nov. 1881, APFR, SRC-ASC, 27 (1871-81), f.1323r.
} 
chevêque de s'impliquer personnellement d'une manière beaucoup plus forte. Elle tourne, en effet, autour de la mise en application d'une décision pontificale.

En décembre 1882, Taschereau délègue à Rome $\mathrm{M}^{\mathrm{gr}}$ Dominique Racine pour faire accélérer l'étude et le règlement des nombreux dossiers qui sont en suspens. L'évêque de Chicoutimi a aussi, dans ses bagages, une nouvelle supplique demandant le démembrement du diocèse de TroisRivières. Le 17 décembre, il écrit au pape pour lui souligner «que le temps est venu de diviser le diocèse de Trois-Rivières et de former le diocèse de Nicolet, et cela dans l'intérêt de $\mathbf{M}^{\mathrm{gr}}$ Laflèche lui-même»; le même jour, il présente les documents nicolétains à la Propagande et, après avoir résumé les arguments favorables, il conclut: «Pour toutes ces raisons je crois donc devoir recommander instamment l'érection du diocèse de Nicolet» ${ }^{24}$. Calixte Marquis, qui est également à Rome, se charge des opérations auprès des cardinaux et des officiers de la Congrégation. À la surprise générale, le problème n'est même pas envoyé au Québec pour connaître le sentiment des évêques. Le 18 février 1883, dans une audience accordée au préfet de la Propagande, Léon XIII affirme qu'il juge que le temps est venu de diviser le diocèse de Trois-Rivières et de créer celui de Nicolet et il demande à la Sacrée Congrégation de lui faire rapport (positio) sur le sujet à l'occasion d'une réunion plénière; le 8 mars suivant, le cardinal Simeoni demande à l'archevêque et à ses collègues de lui faire parvenir des renseignements sur la façon de faire cette division, les frontières du nouveau diocèse et les noms de trois candidats possibles ${ }^{25}$.

Cette décision arrive à Québec au moment où on trouve l'archevêque bien lent à donner son appui au projet déposé à Rome. Il trouverait la façon de procéder peu orthodoxe, mais, rétorque Marquis,

après s'être prononcé pour Nicolet, après avoir enjambé une irrégularité bien plus robuste que celle qui l'arrête aujourd'hui le voilà qui va se déjuger et faire comme $\mathrm{M}^{\mathrm{gr}}$ Laflèche, retirer sa signature!! [...] Le St-Siège veut se débarrasser de $\mathrm{M}^{\mathrm{gr}}$ Laflèche; il suggère le moyen: et voilà l'arch. qui se met de travers dans le chemin!!26

Les récriminations ne cessent pas avec la bonne nouvelle, car on lui reproche désormais de trop tarder à réunir les évêques, ce qui permet à Laflèche d'organiser sa défense et même de prendre le chemin de Rome.

${ }^{24}$ L.-S. Malo, Supplique au Saint Siège pour demander l'érection du diocèse de Nicolet, 6 août 1881, APFR, SOCG, 1021 (1884), f.772r-783r; M $^{\mathrm{gr}}$ D. Racine à Léon XIII, 17 déc. 1882, ASN, Boîte $\mathrm{n}^{\circ}$ 1-DD; le même au card Simeoni, 17 déc. 1882, ibid.

25 Card. Simeoni à $\mathbf{M}^{\mathrm{gr}}$ Taschereau, 8 mars 1883, APFR, Lettere, 379 (1883), f.117v-118r.

${ }^{26} \mathrm{C}$. Marquis à $\mathrm{M}^{\mathrm{gr}} \mathrm{D}$. Racine, 5 fév. 1883, AETR, Fonds Laflèche, B3D30. 
Sa stratégie est pourtant très simple: il considère la création du diocèse de Nicolet comme un fait acquis et il s'emploie à faire mettre à exécution, le plus tôt possible, la décision romaine. Il en fait désormais une question personnelle, car un recul de Rome à propos de Nicolet pourrait entraîner une semblable remise en question des autres dossiers chauds, dont la question universitaire. Et cela, Taschereau ne peut le permettre. Il s'emploie donc de toutes les façons à faire aboutir le décret du 18 février 1883.

Parmi les innombrables démarches faites par l'archevêque, quelquesunes ont un retentissement considérable. Le 19 avril 1883, par exemple, dans un communiqué aux journaux, il rend publique et interprète la décision papale:

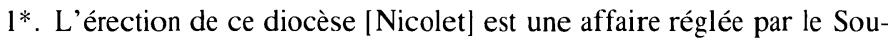
verain Pontife. $2^{*}$. Nos Seigneurs les évêques de la province sont chargés, avec Monseigneur l'Archevêque, de proposer au St-Siège les limites qu'il convient d'y donner et les noms des trois candidats. Sur ce dernier point en particulier, vous êtes prié de ne reproduire aucune rumeur. ${ }^{27}$

C'est une façon de prévenir une volte-face romaine: «la chose étant devenue du domaine public, souligne l'abbé Louis-Nazaire Bégin, Rome ne reviendra certainement pas sur sa décision, malgré toutes les tirailleries et tout le tapage qu'on pourra faire ${ }^{28}$.

D'autre part, Taschereau s'emploie activement à contrecarrer toutes les initiatives de Laflèche ou les manoeuvres pour ouvrir de nouveau le dossier. Dès le début d'avril 1883, il s'organise pour envoyer un message indirect à Trois-Rivières; il dit, au curé Philippe-Hippolyte Suzor de Nicolet, venu le consulter, «qu'il ne fallait pas songer à faire la moindre opposition; [...] que le décret du Souv. Pontife était définitif et absolu et que toute tentative de résistance serait extrêmement mal vue à Rome» ${ }^{29}$. Il ne laisse passer aucun des arguments apportés par l'évêque de Trois-Rivières ou ses partisans; il surveille la réfutation faite à Nicolet et donne de fréquents conseils par l'intermédiaire du grand vicaire Légaré ou de l'abbé Bégin; il envoie à Rome lettres et mémoires qui critiquent chacun des points ou des avancés de Trois-Rivières. Il présente chacun des gestes de Laflèche comme une désobéissance ou un affront au Saint-Siège et un germe de discorde dans le pays. Deux exemples suffiront à l'illustrer. À la fin d'avril 1883, Laflèche décide de retourner à Rome pour défendre l'inté-

27 C.-A. Marois, secr. à l'éditeur, 9 avril 1883, La Vérité, 14 avril 1883, p. 2.

${ }^{28}$ L.-N. Bégin à J.-A.-I. Douville, 14 avril 1883. ASN, Boîte n 1, AG-23.

${ }^{29} I d$. à id., 10 avril 1883 , ibid. 
grité de son diocèse. Dès qu'il apprend la nouvelle, Taschereau envoie un télégramme au cardinal Simeoni pour le mettre en garde, puis il lui écrit une longue lettre: il lui dit que l'évêque de Trois-Rivières a annoncé son départ dans sa circulaire du 24 avril et qu'il a parlé d'en appeler du pape mal renseigné au pape mieux renseigné («clare dicit se a Papa ignorante ad Papam edoctum appellare contra sententiam divisionis dioecesis Trifluvianae») - en fait, Laflèche ne parle pas de voyage et écrit: «Les renseignements si précis que vous me donnez [...] me seront de la plus grande utilité pour renseigner sûrement l'autorité qui doit en juger ${ }^{30}$-; il présente la consultation que Laflèche a faite auprès de son clergé comme un plébiscite plus ou moins libre et fait aussi bien auprès des laïques que des prêtres; enfin, il revient sur les principaux arguments en faveur de la création d'un diocèse à Nicolet et il supplie les autorités romaines de mettre le plus tôt possible à exécution leur décision du 18 février $^{31}$. Au début de 1884 , la création d'une réserve forestière dans le Saint-Maurice apporte un élément nouveau dans le dossier et favorise la position de Laflèche. Taschereau télégraphie immédiatement à Marquis, à Rome, pour le convaincre d'y demeurer encore quelque temps; il lui expédie de la documentation pour qu'il agisse immédiatement auprès de la Propagande et lui-même rédige un mémoire sur le sujet ${ }^{32}$.

Quand la question de la division est remise entre les mains du nouveau délégué apostolique, dom Henri Smeulders, Taschereau supervise le scénario d'intervention des évêques amis et surtout des représentants du Séminaire de Nicolet et tous les documents passent d'abord par ses mains. Lui-même rencontre plusieurs fois le délégué et, quand les choses traînent en longueur et que circule la rumeur que Smeulders aurait fait un rapport défavorable aux Nicolétains, l'archevêque va le rencontrer immédiatement à Montréal. Voyage inutile, puisque, au début d'avril, arrive une nouvelle foudroyante que rapporte le grand vicaire Légaré: « $\mathrm{M}^{\mathrm{gr}}$ Marquis écrit à $\mathrm{M}^{\mathrm{gr}}$ L'Arch. qu'il sait, d'une manière certaine, que le rapport du Com. Apost. est défavorable à Nicolet et à l'Un. Laval» ${ }^{33}$.

$\overline{30} \mathrm{M}^{\mathrm{gr}}$ Laflèche, «Circulaire au clergé», 24 avril 1883, METR, $M^{p r}$ Laflèche, III, pp. 22-23.

${ }^{31} \mathrm{M}^{\mathrm{er}}$ Taschereau au card. Simeoni, 3 mai 1883, APFR, SRC-ASC, 26 (1875-1886), f. $525 \mathrm{r}-526 \mathrm{v}$.

32 C. Marquis, Notes sur la Réserve forestière faite par le Gouvernement de la Province de Québec, dans le territoire du S. Maurice, et qui affecte les trois quarts du diocèse de Trois-Rivières, 9 janv. 1884, ibid., 26, f.677r-778v; M ${ }^{\mathrm{gr}}$ Taschereau, Mémoire concernant les limites assignées à la réserve forestière faite dans le diocèse des TroisRivières en janvier 1884 - Commentaire sur l'édit qui promulgue la réserve forestière. 26 janv. 1884, ibid., 26, f.679r-682r.

33 C.-E. Légaré à J.-A.-I. Douville, 9 avril 1884, ASN, Boîte nº 1, AG-11. 
Taschereau réagit vivement et prend la question en mains. Faisant un pied de nez à Smeulders, qui perd la raison même de sa mission, l'archevêque part pour Rome pour exiger que les problèmes soient de nouveau déférés à la Propagande. Dès son arrivée dans la Ville Éternelle, il mène le combat à pas de charge: audiences chez le pape, visites aux cardinaux, lettres et mémoires à la Propagande, interventions multiples auprès du cardinal Simeoni pour accélérer la discussion en congrégation générale, etc. Il résume toute sa pensée dans un Cenno (Notice) qu'il distribue et commente généreusement ${ }^{34}$. Là encore, il ne laisse passer aucun texte ou aucune démarche de Laflèche et de son procureur Luc Désilets et il les commente ou les fustige. Apprenant, par exemple, que l'évêque de Trois-Rivières a annoncé le règlement de la question en sa faveur et que des adresses de félicitations se signent dans les paroisses du diocèse de Trois-Rivières, Taschereau les présente à la Propagande comme un nouveau «plébiscite contre la division $»^{35}$. Après plusieurs mois de tergiversations - «Santa patienza», s'exclame l'archevêque dans ses lettres - les cardinaux se réunissent le 30 septembre 1884 et ont beaucoup de difficulté à s'entendre: quatre se disent en faveur de la division du diocèse de Trois-Rivières et demandent de rédiger immédiatement le décret requis et de désigner le premier évêque de Nicolet; un autre appuie le démembrement, mais suggère de ne pas le faire avant l'envoi d'un nouveau délégué au Canada; trois opinent qu'à cause de circonstances nouvelles, il vaut mieux différer la division et se contenter de recommander le Séminaire de Nicolet à la bienveillance de l'évêque de Trois-Rivières, tout en lui demandant un rapport annuel sur la question. À son audience du 5 octobre suivant, Léon XIII opte pour la position médiane:

Le principe de la division du diocèse est maintenu; mais cette division ne devra pas se faire avant qu'un nouveau Commissaire Apostolique ne soit envoyé dans la province du Canada. ${ }^{36}$

Cette victoire termine d'une certaine façon le travail de Taschereau dans ce dossier. Il publie néanmoins la décision romaine, ce qui lui attire une fois de plus une dénonciation de Laflèche qui l'accuse de l'avoir fait «contrairement à la recommandation» du cardinal préfet et d'avoir altéré le texte romain en ne parlant pas de la venue d'un nouveau délégué ${ }^{37}$. De

\footnotetext{
${ }^{34} \mathrm{M}^{\mathrm{gr}}$ Taschereau, Cenno delle ragioni che stanno in favore della divisione della Diocesi "des Trois-Rivières» e della creazione di une nuova sede Épiscopale à Nicolet, mai 1884 , APFR, SOCG, 1021 (1884), f.863r-865v.

${ }^{35} \mathrm{M}^{\mathrm{gr}}$ Taschereau à $\mathrm{M}^{\mathrm{gr}} \mathrm{Jacobini}, 21$ juin 1884, ibid., SRC-ASC, 23 (1883-1884), f. 1006r-1006v.

${ }^{36}$ Card. Pitra, [Procès-verbal de la congrégation générale de la Propagande], 10 oct. 1884, ibid., Acta, 253 (1884), f.605r.

${ }^{37} \mathrm{M}^{\mathrm{gr}}$ Laflèche au card Simeoni, 21 janv. 1885, ibid., SRC-ASC. 26, f.924r-926v.
} 
même, il ne néglige pas de renseigner le nouveau délégué. $\mathrm{M}^{\mathrm{gr}} \mathrm{J}$ ohn Cameron, évêque d'Arichat, qui est bien connu à Québec et est, dit Luc Désilets, «l'ami intime des adversaires de mon Évêque» ${ }^{38}$. Enfin, il surveille de très près la situation et quand, par exemple, court la rumeur d'un prochain départ de Laflèche pour Rome, il télégraphie immédiatement à Simeoni qui lui répond par la même voie: «il n'est pas opportun que Laflèche vienne à Rome: qu'il s'en abstienne». Ce qui lui vaut cette riposte de l'évêque de Trois-Rivières: "Quant au télégramme que Votre Grandeur a provoqué de Rome sur un simple cancan, je laisse à votre conscience à juger devant Dieu ce qu'il faut penser d'une telle manière d'agir» ${ }^{39}$. Et ce commentaire acerbe qui est une sorte de jugement global sur tout le travail que l'archevêque a fait pour la création du diocèse de Nicolet:

De quel droit le Métropolitain vient-il ainsi me barrer le chemin de Rome, lui qui l'année dernière a tourné le dos au Commissaire Apostolique au Canada pour aller travailler à ruiner auprès du St-Siège sa mission bienfaisante ici? Et $\mathrm{M}^{\mathrm{gr}}$ de Chicoutimi? N'y aura-t-il donc qu'au défenseur de la vérité et de la justice, qui a pour lui les jugements favorables des employés du St-Siège, que l'on barrera ainsi le chemin de Rome pour l'empêcher de remplir les devoirs les plus sacrés de la charge épiscopale? Et les étrangers, les agresseurs injustes seront-ils les seuls qui auront toujours leurs coudées franches? ${ }^{40}$

\section{Conclusion}

Ce survol trop rapide prouve amplement, il me semble, la participation régulière et efficace de $\mathbf{M}^{\mathrm{gr}}$ Taschereau aux démarches qui ont mené à la création du diocèse de Nicolet. Pendant de longues années de discussions et de manoeuvres de toutes sortes, il s'est toujours rangé du côté des partisans de la nouvelle circonscription ecclésiastique et a fait peser en leur faveur le poids de son influence et de son réseau d'amis à Rome. Dans la dernière phase de la question, son intervention auprès des instances romaines a été déterminante pour un règlement immédiat et définitivement favorable aux Nicolétains. Est-ce que cela en fait le principal artisan de la création du diocèse de Nicolet, plus que $\mathbf{M}^{\mathrm{gr}}$ Marquis, plus que les prêtres du Séminaire? Je ne saurais le dire et je me contente de souligner que l'intervention de l'archevêque a été soutenue, importante, essentielle et, à un moment donné, déterminante.

\footnotetext{
${ }^{38}$ Luc Désilets au card. Simeoni, 8 mai 1885, ibid., 26, f.990r-990v.

${ }^{39} \mathrm{M}^{\mathrm{gr}}$ Laflèche à $\mathrm{M}^{\mathrm{gr}}$ Taschereau, 27 mai 1885, cité dans Robert Rumilly, Monseigneur Laflèche et son temps, Montréal, Simpson, 1945, p. 278.

${ }^{40} \mathrm{M}^{\mathrm{gr}}$ Laflèche à L. Désilets, 29 mai 1885, AETR. Fonds Laflèche, B3D24.
} 
Une autre remarque m'apparaît aussi importante: le problème du démembrement du diocèse de Trois-Rivières et de la création de celui de Nicolet n'est pas isolé et il ne se comprend pas sans connaître les autres débats qui ont cours à l'époque. Il n'est qu'une facette de cette lutte de pouvoir qui met aux prises les intransigeants réunis autour de Laflèche et les modérés qui suivent Taschereau. Et la question universitaire est comme l'épicentre de toutes ces querelles qui secouent l'épiscopat et le clergé du Québec. Et c'est cette question qui explique le mieux l'implication de l'archevêugue dans le dossier de Nicolet: diviser le diocèse de Trois-Rivières, c'est affaiblir celui qui a pris la relève de $\mathrm{M}^{\mathrm{gr}}$ Bourget à la tête des partisans d'une université autonome à Montréal; obliger Laflèche à accepter le décret romain, c'est à la fois lui faire reconnaître sa défaite et peut-être le pousser à démissionner, et prévenir toute velléité de revenir sur la décision romaine de 1876 d'ouvrir une succursale de l'Université Laval à Montréal. Voilà pourquoi il n'a pas été difficile à Calixte Marquis de «faire faire» le diocèse de Nicolet par son ami l'archevêque Taschereau. 